\title{
Phenotypic analysis of familial breast cancer: Comparison of BRCAx tumors with BRCA1-, BRCA2-carriers and non-familial breast cancer
}

\author{
F. Aloraifi ${ }^{\mathrm{a}, \mathrm{b}, *}$, M. Alshehhi ${ }^{\mathrm{c}}, \mathrm{T}^{\mathrm{T}}$ McDevitt $^{\mathrm{c}}, \mathrm{N} \cdot \operatorname{Cody}^{\mathrm{c}}, \mathrm{M}$. Meany ${ }^{\mathrm{c}}$, \\ A. O’Doherty ${ }^{\mathrm{a}, \mathrm{d}}$, C.M. Quinn ${ }^{\mathrm{a}, \mathrm{d}}$, A.J. Green ${ }^{\mathrm{a}, \mathrm{c}}$, A. Bracken ${ }^{\mathrm{b},{ }^{*} *}$, \\ J.G. Geraghty ${ }^{\mathrm{a}, \mathrm{d}}$ \\ ${ }^{a}$ School of Medicine and Medical Science, University College Dublin, Ireland \\ ${ }^{\mathrm{b}}$ Smurfit Institute of Genetics, Trinity College Dublin, Ireland \\ ${ }^{\mathrm{c}}$ National Centre for Medical Genetics, Ireland \\ ${ }^{\mathrm{d}}$ St. Vincent University Hospital, Ireland
}

Accepted 15 January 2015

Available online

\begin{abstract}
Aims: Women with inherited pathogenic mutations in the BRCA1 or BRCA2 genes have up to an $85 \%$ risk of developing breast cancer in their lifetime. However, only about $20 \%$ of familial breast cancer is attributed to mutations in BRCA1 and BRCA2, while a further 5-10\% are attributed to mutations in other rare susceptibility genes such as TP53, STK11, PTEN, ATM and CHEK2. Despite extensive efforts to explain the missing heritability of this disease, the majority of familial clustering in breast cancer remains largely unexplained. We aim to analyze the pathology of familial cases of which no pathogenic mutation is yet identified.

Methods: We compared the pathological phenotype of BRCA1/BRCA2 negative familial breast cancer (BRCAx) to BRCA1-positive, BRCA2-positive and sporadic cases without a family history. Age-adjusted analysis is summarized in odd's ratios and confidence intervals for tumor type, grade, lymph node, ER and HER2 status.

Results: We found non-familial cases to be more likely to be ER positive $(P=0.041)$ as compared with BRCAx tumors. More cases of lobular carcinoma were found with BRCAx as compared to BRCA1 tumors $(P=0.05)$. After multivariate logistic regression analysis, BRCAx tumors are more likely ER positive $(P=0.001)$ and HER2 positive $(P=0.047)$ in comparison to BRCA1. Conversely, BRCAx cases are less likely to be ER positive $(P=0.02)$ but more likely to be HER2 positive $(P=0.021)$ as compared with BRCA2 tumors. Conclusion: Our findings suggest that BRCA1, BRCA2 and BRCAx tumors differ in phenotype from non-familial and familial BRCA1positive and BRCA2-positive tumors. Further studies will need to be performed in this important population in order to develop strategies for early detection and prevention.
\end{abstract}

(C) 2015 Elsevier Ltd. All rights reserved.

Keywords: Breast cancer; BRCA1 gene; BRCA2 gene; Selective estrogen receptor modulators; Genetics; Chemoprevention

Abbreviations: BRCAx, non-BRCA1 or non-BRCA2 familial breast cancer; ER, estrogen receptor; HER2, human epidermal growth factor receptor 2 receptor; MLPA, multiplex ligation-dependent probe amplification; NCMG, Irish National Centre for Medical Genetics; CI, confidence interval; OR, Odds ratio; DCIS, ductal carcinoma in situ; IDC, invasive ductal carcinoma; ILC, invasive lobular carcinoma.

* Corresponding author. Smurfit Institute of Genetics, Trinity College Dublin, Ireland. Tel.: +353 (1) 8961908; fax: +353 (1) 6798558 .

** Corresponding author.

E-mail address: faloraifi@rcsi.ie (F. Aloraifi).

\section{Introduction}

It is well recognized that women who have a pathogenic mutation in either BRCA1 or BRCA2 genes have up to a $68-88 \%$ increased risk of developing breast cancer. ${ }^{1}$ These patients are offered risk-reducing measures such as intensive radiological screening and prophylactic surgery. ${ }^{2,3}$ However, the frequency of familial breast cancer cases attributed to mutations in BRCA1 and BRCA2 vary from only 12.5 to $31 \%$ in studies on a large series of 
patients of multiple ethnicities. ${ }^{4,5}$ Therefore, the majority of familial cases have been defined as BRCAx (a term that has been used to describe familial breast cancer cases found to be negative for those two genes). Although other susceptibility genes such as TP53, STK11, PTEN, ATM, PALB2 and $C H E K 2$ have since been identified, they still do not explain the majority of cases. ${ }^{6-12}$

While previously those at high-risk would be restricted to screening for BRCA1 and BRCA2 genes, developments in sequencing technologies have made it possible to test for multiple genes at lower cost, specifically using multigene panel testing with next-generation sequencing. Therefore, much like the efforts that have been made to describe $B R C A 1$ and BRCA2 tumor phenotypes in order to estimating the chance to identifying new pathogenic mutations, characterizing the BRCAx phenotype may shed light on our understanding of the majority of the familial clustering that is yet to be explained.

Specific protein characteristics and tumor histology have been described in patients with pathogenic mutations in $B R C A 1$ and BRCA2. Studies have shown that mutation carriers do not necessarily all have the same phenotype. ${ }^{13,14}$ Certain histological patterns have been demonstrated especially in BRCA1 mutation carriers. ${ }^{13,15-18}$ Triple negativity, i.e. lacking expression of estrogen, progesterone, and Her2 receptors, and high histological grade tend to be common amongst BRCA1 mutation carriers. ${ }^{13,15-18}$ On the other hand, BRCA2 tumor histology seems to be more heterogeneous and similar to the common breast cancer pattern being intermediate grade, luminal B and estrogen/progesterone positive. ${ }^{16-20}$

Whilst the phenotypes of BRCA1 and BRCA2 have been reasonably well described, studies on BRCAx tumors, which make up the majority of familial cases are somewhat limited. Lakhani and colleagues compared BRCA1, BRCA2, BRCAx and sporadic breast cancer and noted that non-BRCA1/2 breast cancers were of significantly lower grade. ${ }^{15}$ Palacious et al. observed similar features between these tumor types in Spanish samples. ${ }^{20}$ These studies categorized BRCAx cases as having a more favorable long-term outcome than average. ${ }^{21}$ In this current study, we present and analyze the associated phenotype of BRCAx tumors as compared to BRCA1, BRCA2 and non-familial tumors screen-detected through mammography.

\section{Methods}

\section{Ethical approval}

Permission for this study was obtained from the Research and Ethics Committee at Our Lady's Children's Hospital, Crumlin, Dublin, in the Republic of Ireland.

Samples

The familial groups, i.e. BRCAx $(\mathrm{N}=209)$, BRCA1 $(\mathrm{N}=63)$ and BRCA2 $(\mathrm{N}=60)$ groups consisted of patients referred from 2007 to 2011 to the Irish National Centre for Medical Genetics (NCMG) for genetic counseling and testing. Cases were included on the basis of having a personal history of breast cancer, a Manchester score of $>16$, at least 2 other affected relatives or an affected relative and bilateral breast cancer, since both these features increase the likelihood of a familial predisposition (and the risk of developing breast cancer by 4 -fold). All cases were screened for variants in the $B R C A 1$ or $B R C A 2$ genes by bidirectional sequencing and multiplex ligationdependent probe amplification (MLPA). This analysis was performed externally at the West Midlands Regional Genetics Centre (Birmingham, United Kingdom). All patients were unrelated and asked to complete a detailed questionnaire, which required them to provide demographic details, cancer and family history of breast cancer for the previous three generations. A limited number of probands were subsequently contacted to clarify some of their clinical details.

Screen-detected breast cancer geographically-matched cases $(\mathrm{N}=670)$ were obtained from the BreastCheck Merrion Unit, Dublin, Ireland database, which archives screendetected patients with breast cancer from the years 2009-2011. BreastCheck invites females 50-64 for mammographic screening and thus these cases were discovered through mammography.

\section{Histology review}

Histological reports from the respective hospitals were collected for each familial case. Histological type, grade, stage, receptor status (ER, PR, Her2) was obtained for each case. In some instances, initial excision of the tumor was performed a significant time before referral to the NCMG and thus full histology reporting was incomplete; hence we had to exclude some index cases. However, $100 \%$ of the histology reports were successfully obtained from the screen-detected cohort.

\section{Statistical methods}

Statistical tests in this study were done with Stata version 12.0 (Stata Corporation, College Station, TX). We tested dichotomous variables with either Pearson's chi-2 statistic or Fisher's exact test. We tested continuous variables (tumor grade) with a t-test and Wilcoxon-Mann-Whitney test (a model which assumes they are from the same normal distribution). To assess multivariate analysis, we used a logistic regression analysis. We considered a p-value of 0.05 or less as significant. All pvalues are two-sided.

\section{Results}

To examine the features of multiple-case BRCAx tumors, histological data from 332 familial (209 BRCAx, 63 BRCA1, 60 BRCA2 tumors) and 670 non-familial breast 
Table 1

Demographics of BRCAx, BRCA1, BRCA2 and Non-familial cases included in the study. In age, asterisk* denotes that age is displayed in mean (min-max). DCIS = Ductal carcinoma in situ, IDC = Invasive ductal carcinoma, ILC = Invasive lobular carcinoma, ER $=$ Estrogen receptor, HER2 $=$ human epidermal growth factor receptor 2 receptor.

\begin{tabular}{|c|c|c|c|c|}
\hline & $\operatorname{BRCAx}(\mathrm{N}=209)$ & BRCA1 $(\mathrm{N}=63)$ & BRCA2 $(\mathrm{N}=60)$ & Non-familial $(\mathrm{N}=670)$ \\
\hline \multicolumn{5}{|c|}{ Age of diagnosis* } \\
\hline & $42(22-64)$ & $40(24-60)$ & $44(20-69)$ & $57(48-65)$ \\
\hline \multicolumn{5}{|c|}{ Cancer type } \\
\hline DCIS & $19(10.44 \%)$ & $4(6.90 \%)$ & $4(7.02 \%)$ & $124(18.51 \%)$ \\
\hline IDC & $137(75.27 \%)$ & $51(87.93 \%)$ & $46(80.70 \%)$ & $450(67.16 \%)$ \\
\hline ILC & $21(11.54 \%)$ & $1(1.72 \%)$ & $4(7.02 \%)$ & $74(11.04 \%)$ \\
\hline Mixed & $3(1.65 \%)$ & $2(3.45 \%)$ & $3(5.26 \%)$ & $15(2.23 \%)$ \\
\hline \multicolumn{5}{|l|}{ Grade } \\
\hline 1 & $16(10.12 \%)$ & $3(5.66 \%)$ & $3(5.36 \%)$ & $142(21.23 \%)$ \\
\hline 2 & $70(44.30 \%)$ & $15(28.30 \%)$ & $23(41.07 \%)$ & $305(45.60 \%)$ \\
\hline 3 & $72(45.57 \%)$ & $35(66.04 \%)$ & $30(53.57 \%)$ & $222(33.18 \%)$ \\
\hline \multicolumn{5}{|c|}{ Lymph node status } \\
\hline & $92(51.69 \%)$ & $68(38.20 \%)$ & $34(62.96 \%)$ & $149(22.23 \%)$ \\
\hline \multicolumn{5}{|c|}{ Receptor Status } \\
\hline ER & $130(70.65 \%)$ & $19(36.54 \%)$ & $45(78.95 \%)$ & $549(87.7 \%)$ \\
\hline Her2 & $40(21.86 \%)$ & $5(9.43 \%)$ & $6(10.53 \%)$ & $65(12.31 \%)$ \\
\hline
\end{tabular}

tumors were collected for this study. Cohort features are listed in detail in Table 1. The phenotypic profiles of BRCAx tumors were established by analyzing tumor type, grade and receptor status and comparing them with BRCA1, BRCA2 and screen-detected tumors. Statistical testing was adjusted for age (Table 2).

\section{Non-Familial Breast Cancer Group}

The non-familial breast cancer cohort included 670 breast cancer cases diagnosed by mammography through a national screening program in Ireland (BreastCheck). Not surprisingly, non-familial tumors were more likely to be DCIS with an Odds Ratio (OR) of 0.31 , a 95\% Confidence interval (CI) from 0.15 to 0.67 and an associated $\mathrm{P}$ value of $\mathrm{P}=0.003$. They were also less likely to have positive lymph nodes (OR 2.44, 95\% CI 1.46 to 4.07, $\mathrm{P}=0.001)$ and more likely ER receptor positive than the BRCAx tumors (OR $0.52,95 \%$ CI 0.29 to $0.98, \mathrm{P}=0.041$ ).

\section{BRCA1-Positive Familial Breast Cancer Group}

Interestingly, the likelihood of lobular carcinoma within the BRCAx cohort was higher than that of the BRCA1 cohort (OR 7.3, 95\% CI 0.95-56.10, $\mathrm{P}=0.050$ ). BRCA1 tumors were also of higher grade than BRCAx. After controlling for other factors, the multivariate regression results showed that BRCAx tumors remained more likely receptor positive as compared with $B R C A 1$ : ER receptor showed an OR of 3.83 (CI 95\% 1.76-8.34, P =0.001) and HER2 receptor showed an OR of 2.99 (95\% CI $1.01-8.87, \mathrm{P}=0.47$ ).

Table 2

Uni-variate statistical analysis comparing BRCAx familial tumors to BRCA1, BRCA2 and nonfamilial tumors. DCIS = Ductal carcinoma in situ, $\mathrm{IDC}=$ Invasive ductal carcinoma, ILC $=$ Invasive lobular carcinoma, ER $=$ Estrogen receptor, HER2 = human epidermal growth factor receptor 2 receptor, $\mathrm{OR}=$ Odds ratio, $\mathrm{CI}=$ confidence interval.

\begin{tabular}{|c|c|c|c|c|c|c|c|c|c|}
\hline & \multicolumn{3}{|c|}{ BRCAx vs $B R C A 1$} & \multicolumn{3}{|c|}{ BRCAx vs $B R C A 2$} & \multicolumn{3}{|c|}{ BRCAx vs non-familial } \\
\hline & OR & $95 \% \mathrm{CI}$ & $P$ value & OR & $95 \% \mathrm{CI}$ & $P$ value & OR & $95 \% \mathrm{CI}$ & $P$ value \\
\hline \multicolumn{10}{|l|}{ Type } \\
\hline DCIS & 1.68 & $0.54-5.23$ & 0.365 & 1.39 & $0.45-4.32$ & 0.567 & 0.31 & $0.15-0.67$ & 0.003 \\
\hline IDC & 0.42 & $0.18-0.99$ & 0.048 & 0.76 & $0.36-1.59$ & 0.465 & 1.43 & $0.84-2.44$ & 0.185 \\
\hline ILC & 7.3 & $0.95-56.10$ & 0.050 & 1.84 & $0.60-5.70$ & 0.289 & 1.68 & $0.83-3.42$ & 0.148 \\
\hline Mixed & 0.47 & $0.08-2.89$ & 0.414 & 0.30 & $0.06-1.53$ & 0.148 & 0.89 & $0.195-4.02$ & 0.877 \\
\hline \multicolumn{10}{|l|}{ Grade } \\
\hline 1 & 0.38 & $0.21-0.70$ & 0.002 & 0.59 & $0.35-1.00$ & 0.050 & 1.09 & $0.71-1.68$ & 0.679 \\
\hline \multicolumn{10}{|l|}{2} \\
\hline \multicolumn{10}{|l|}{3} \\
\hline \multicolumn{10}{|c|}{ Lymph node status } \\
\hline & 0.94 & $0.51-1.71$ & 0.830 & 0.48 & $0.25-0.91$ & 0.025 & 2.44 & $1.46-4.07$ & 0.001 \\
\hline \multicolumn{10}{|c|}{ Receptor Status } \\
\hline ER & 4.21 & $2.17-8.14$ & $<0.0001$ & 0.55 & $0.26-1.17$ & 0.119 & 0.52 & $0.29-0.98$ & 0.041 \\
\hline HER2 & 2.70 & $1.01-7.27$ & 0.049 & 2.24 & $0.89-5.64$ & 0.085 & 1.37 & $0.70-2.68$ & 0.362 \\
\hline
\end{tabular}




\section{BRCA2-Positive Familial Breast Cancer Group}

In comparison to BRCAx, BRCA2 tumors showed an aggressive phenotype of being of higher grade (OR 0.59, $\mathrm{P}=0.05)$ and more likely to have positive lymph nodes (OR $0.48,95 \%$ CI $0.25-0.91, \mathrm{P}=0.025$ ). However, multivariate logistic regression results revealed that BRCAx tumors remained less likely to be ER positive (OR 0.35, $95 \%$ CI $0.14-0.85, \mathrm{P}=0.020)$ and more likely HER2 positive (OR 3.21, 95\% CI 1.19-8.64, P = 0.21).

\section{Discussion}

By analyzing histological data from familial nonBRCA1/2 (BRCAx) with BRCA1-positive, BRCA2positive and non-familial screen-detected breast cancer tumors, we report that BRCAx tumors are, in severity, between that of familial and non-familial cases. In particular, BRCAx receptor status is less likely ER positive than nonfamilial cases and after multivariate logistic regression analysis is more likely ER and HER2 positive as compared with BRCA1 tumors, and less likely ER positive and more likely HER2 negative as compared with BRCA2 tumors. We found that not only do BRCAx tumors have a trend towards a different histological profile than that of nonfamilial cases, but they generally also seem to have a more aggressive phenotype. This is in contrast to a the findings of a small number of previous studies that compared the BRCAx familial phenotype with sporadic breast cancer. ${ }^{15}$

In an attempt to characterize the phenotype of likely mutation carriers for clinical testing, several key studies aimed to examine BRCA1, BRCA2 and sporadic breast cancer phenotypes. ${ }^{13,22-24}$ While BRCAl tumors tend to be of high grade and triple negative, some have reported that BRCAx tumors are of lower grade, even compared to sporadic cases. However, their cohort selection was based on symptomatic sporadic cases. ${ }^{15}$ They further commented that their results need to be interpreted with caution as biases may exist due to hereditary cancers being diagnosed early and may therefore appear to be of lower grade. Keeping that in mind, we decided to compare the phenotype of screen-detected breast tumors with familial cases to try and reduce that bias.

Similarly, the results of our study may be subject to other types of bias. Firstly, the BRCAx cohort was selected on the basis of having a personal history of breast cancer and presence of family history, while the screen-detected cohort was identified through a national screening program from the age of 50 through mammographic screening. The difference in the age of onset between the two groups may of course contribute to the differences we see in tumor stage. Nonetheless, we do not expect receptor status to be affected by this bias, since it is in essence representative of the tumor features regardless of disease progression. In addition, our observation that DCIS is more common in the non-familial than the familial cohort is inevitable since DCIS is more common in a screened rather than a nonscreened cohort as DCIS rarely resents symptomatically. This bias can similarly be extended to lymph node status.

Preventative strategies for $B R C A 1$ and $B R C A 2$ carriers are relatively well-established and have been predominantly focused on surgical strategies, such as bilateral mastectomy and reducing estrogen exposure by bilateral salpingo-oophrectomy. ${ }^{3,25,26}$ As breast cancer risk may be as high as $85 \%$ in $B R C A 1$ and $B R C A 2$ carriers, surgical intervention can be a good option to reduce risk. ${ }^{1}$ The clinical management is not as clear cut for unaffected members of BRCAx families for whom breast cancer risk is relatively unknown.

Chemoprevention in BRCA1/2 carriers is controversial as tumors generally have differing hormonal phenotypes, especially in BRCAl mutation carriers for whom the tumor phenotype is typically basal-like and thus triple negative. ${ }^{27}$ Selective estrogen response modifiers (or SERMs) such as Tamoxifen and Raloxifene are the gold-standard for treating both early and advanced hormone receptor-positive breast cancer. ${ }^{28,29}$ Studies have shown their extended usefulness in breast cancer prevention in high-risk individuals. ${ }^{30}$ The NSABP trial showed a $43 \%$ reduction in invasive breast cancer in high-risk women taking Tamoxifen, which was only seen in ER-positive cancers. ${ }^{30}$ Data from the randomized primary prevention studies suggested that the benefit of Tamoxifen was confined to the prevention of ER-positive breast cancer. ${ }^{31}$ Surprisingly however, there has been evidence that although BRCA1 tumors are less likely ER positive than BRCA2 tumors, the reduction in risk after Tamoxifen is relatively similar. ${ }^{32,33}$ Although results from our study show that $70 \%$ of BRCAx cases had ER-positive tumors, ER receptor negativity in BRCAx cases was the only independent variable. The usefulness of chemoprevention is therefore questionable and should be carefully assessed in these cases. While Tamoxifen treatment is associated with a $37 \%$ reduction in breast cancer incidence, there are associated risks, such as potentially lethal venous thromboembolic events. ${ }^{34}$ This risk is an important consideration in the case of at risk individuals who have not yet developed breast cancer and in whom ER receptor status is therefore unknown. An alternative would be the results from the MAP-3 trial, which showed a $65 \%$ reduction of invasive breast cancer in postmenopausal women with Aromatase inhibitor Exemestane with minimal side effects at 3 years. ${ }^{35}$ Further studies will need to be performed to define the usefulness of chemoprevention for this important BRCAx familial population.

In conclusion, our study aimed to shed light on the familial BRCAx phenotype through a comparison with BRCA1, BRCA2 and non-familial breast cancer tumors. We have shown that BRCAx tumors are less likely ERpositive than non-familial cases, but more likely ERpositive than BRCA1 and BRCA2 tumors. Results from 
this study can be important when it comes to the management of unaffected members of families with breast cancer clustering found not to carry BRCAl or BRCA2 mutations and further studies will need to be set our and sought for to assess the suitability of chemoprevention in these patients and their unaffected relatives. In addition, the ongoing dramatic improvements in the speed, scale and cost of DNA sequencing through developments in high-throughput methodologies have provided a realistic alternative to GWAS approaches to identify candidate genes, especially to high-risk rare disease variants. ${ }^{36}$ Establishing phenotype-genotype databases can help us further find genetic causes of disease to aim for early diagnosis. ${ }^{37}$

\section{Role of funding source}

None.

\section{Conflict of interest statement}

All authors have no conflicts of interest or financial ties to disclose.

\section{References}

1. Evans DGR, Shenton A, Woodward E, Lalloo F, Howell A, Maher ER. Penetrance estimates for BRCA1 and BRCA2 based on genetic testing in a Clinical Cancer Genetics service setting: risks of breast/ovarian cancer quoted should reflect the cancer burden in the family. BMC Cancer 2008;8:155.

2. Kriege M, Brekelmans CT, Boetes C, et al. Efficacy of MRI and mammography for breast-cancer screening in women with a familial or genetic predisposition. Engl J Med 2004;351:427-37.

3. Hartmann L, Schaid D, Woods J, Crotty T, Myers J. Efficacy of bilateral prophylactic mastectomy in women with a family history of breast cancer. Engl J Med 1999;340(2):77-84.

4. Mann GJ, Thorne H, Balleine RL, et al. Analysis of cancer risk and BRCA1 and BRCA2 mutation prevalence in the $\mathrm{kConFab}$ familial breast cancer resource. Breast Cancer Res 2006;8(1):R12.

5. Hall MJ, Reid JE, Burbidge LA, et al. BRCA1 and BRCA2 mutations in women of different ethnicities undergoing testing for hereditary breast-ovarian cancer. Cancer 2009;115(10):2222-33.

6. Malkin D, Li FP, Strong LC, et al. Germ line p53 mutations in a familial syndrome of breast cancer, sarcomas, and other neoplasms. Science 1990;250(4985):1233-8.

7. Hemminki A, Markie D, Tomlinson T, et al. A serine/threonine kinase gene defective in Peutz-Jeghers syndrome. Nature 1998;114(7):667-8.

8. Rahman N, Seal S, Thompson D, et al. PALB2, which encodes a BRCA2-interacting protein, is a breast cancer susceptibility gene. Nat Genet 2007:39(2):165-7.

9. Seal S, Thompson D, Renwick A, et al. Truncating mutations in the Fanconi anemia J gene BRIP1 are low-penetrance breast cancer susceptibility alleles. Nat Genet 2006;38(11):1239-41.

10. Nelen MR, Padberg GW, Peeters EA, et al. Localization of the gene for Cowden disease to chromosome 10q22-23. Nat Genet 1996;13: 114-6.

11. Broeks A, Urbanus JH, Floore AN, et al. ATM-heterozygous germline mutations contribute to breast cancer-susceptibility. Am J Hum Genet 2000; 66:494-500.
12. Kainu T, Juo SH, Desper R, et al. Somatic deletions in hereditary breast cancers implicate $13 \mathrm{q} 21$ as a putative novel breast cancer susceptibility locus. PNAS 2000;97(17):9603-8.

13. Palacios J, Robles-Frias MJ, Castilla MA, Lopez-Garcia MA, Benitez J. The molecular pathology of hereditary breast cancer. Pathobiology 2008;75:85-94.

14. Vargas AC, Reis-Filho JS, Lakhani SR. Phenotype-genotype correlation in familial breast cancer. J Mammary Gland Biol Neoplasia 2011;16(1):27-40.

15. Lakhani S, Gusterson B. The pathology of familial breast cancer: histological features of cancers in families not attributable to mutations in BRCA1 or BRCA2. Clin Cancer Res 2000;6:782-9.

16. Lakhani SR, Van De Vijver MJ, Jacquemier J, et al. The pathology of familial breast cancer: predictive value of immunohistochemical markers estrogen receptor, progesterone receptor, HER-2, and p53 in patients with mutations in BRCA1 and BRCA2. J Clin Oncol 2002; 20:2310-8.

17. Bane AL, Pinnaduwage D, Colby S, et al. Expression profiling of familial breast cancers demonstrates higher expression of FGFR2 in BRCA2-associated tumours. Breast Cancer Res Treat 2009;117: 183-91.

18. Oldenburg R, Kroeze-Jansema K, Meijers-Heijboer H, et al. Characterization of familial non-BRCA1/2 breast tumours by loss of heterozygosity and immunophenotyping. Clin Cancer Res 2006;12(6): 1693-700.

19. Balleine R, Provan P. Familial concordance of breast cancer pathology as an indicator of genotype in multiple-case families. Genes Chromosomes Cancer 2010;49:1082-94.

20. Palacios J, Honrado E, Osorio A. Immunohistochemical characteristics defined by tissue microarray of hereditary breast cancer not attributable to BRCA1 or BRCA2 mutations differences from. Clin Cancer Res 2003;9:3606-14.

21. Eccles DM, Pichert G. Familial non-BRCA1/BRCA2-associated breast cancer. Lancet Oncol 2005;6(9):705-11.

22. Da Silva L, Lakhani SR. Pathology of hereditary breast cancer. Mod Pathol 2010;(Suppl. 2):S46-51.

23. Loughrey M, Provan PJ, Byth K, Balleine RL. Histopathological features of "BRCAX" familial breast cancers in the kConFab resource. Pathology 2008;40(4):352-8.

24. Eerola H, Heikkilä P, Tamminen A, Aittomäki K, Blomqvist C, Nevanlinna H. Histopathological features of breast tumours in BRCA1, BRCA2 and mutation-negative breast cancer families. Breast Cancer Res 2005;7(1):R93-R100.

25. Domchek SM, Friebel TM, Neuhausen SL, et al. Mortality after bilateral salpingo-oophorectomy in BRCA1 and BRCA2 mutation carriers: a prospective cohort study. Lancet Oncol 2006;7(3):223-9.

26. Meijers-Heijboer H, van Geel B, van Putten WL, et al. Breast cancer after prophylactic bilateral mastectomy in women with a BRCA1 or BRCA2 mutation. N Engl J Med 2001;345(3):159-64.

27. Phillips KA, Lindeman GJ. Breast cancer prevention for BRCA1 and BRCA2 mutation carriers: is there a role for tamoxifen? Future Oncol 2014;10(4):499-502.

28. Fisher B, Costantino JP, Wickerham DL, et al. Tamoxifen for prevention of breast cancer: report of the National Surgical Adjuvant Breast and Bowel Project P-1 Study. J Natl Cancer Inst 1998;90(18):137188.

29. Schechter A, Stern D, Vaidyanathan L. The neu oncogene: an erb-Brelated gene encoding a 185,000-Mr tumour antigen. Nature 1984; 312:513-6.

30. Fisher B, Costantino JP, Wickerham DL, et al. Tamoxifen for prevention of breast cancer: current status of the National Surgical Adjuvant Breast and Bowel Project P-1 Study. J Natl Cancer Inst 2005;97: $1652-62$.

31. Cuzick J, Sestak I, Bonanni B, et al. Selective oestrogen receptor modulators in prevention of breast cancer: an updated meta-analysis of individual participant data. Lancet 2013;381(9880):1827-34. 
32. Metcalfe K, Lynch HT, Ghadirian P, et al. Contralateral breast cancer in BRCA1 and BRCA2 mutation carriers. J Clin Oncol 2004;22(12): 2328-35.

33. Narod SA. Hormonal prevention of hereditary breast cancer. Ann N $Y$ Acad Sci 2001;952:36-43.

34. Cuzick J, Powles T, Veronesi U, et al. Overview of the main outcomes in breast-cancer prevention trials. Lancet 2003;361(9354): 296-300.
35. Goss PE, Ingle JN, Alés-Martínez JE, et al. Exemestane for breastcancer prevention in postmenopausal women. NEJM 2011;364: 2381-91.

36. Mardis ER. A decade's perspective on DNA sequencing technology. Nature 2011;470(7333):198-203.

37. Evans DGR, Lalloo F, Wallace A, Rahman N. Update on the Manchester Scoring System for BRCA1 and BRCA2 testing. J Med Genet 2005; $42: \mathrm{e} 39$. 